\title{
Plasmablastic plasmacytoma of the rectum and anal canal in a patient with ulcerative colitis
}

\author{
Katarina Jankovic ${ }^{1}$, Sanja Rajic ${ }^{1}$, Sanja Dragasevic ${ }^{2}$, Ivan Jovanovic ${ }^{2}$ \\ ${ }^{1}$ Clinic for Gastroenterology and Hepatology, Clinical Centre of Serbia, Belgrade, Serbia \\ ${ }^{2}$ Clinic for Gastroenterology and Hepatology, Clinical Centre of Serbia; School of Medicine, University of Belgrade, Belgrade, Serbia
}

Gastroenterology Rev 2021; 16 (2): 166-169

DOI: https://doi.org/10.5114/pg.2021.106668

\begin{abstract}
Address for correspondence: Prof. Ivan Jovanovic MD, PhD, FASGE, Clinic for Gastroenterology and Hepatology, Clinical Centre of Serbia; School of Medicine, University of Belgrade, Belgrade, Serbia, e-mail: ivangastro@beotel.net
\end{abstract}

Plasma cell neoplasms (PCN) represents a group of tumours originating from abnormal plasma cells, which are further classified into multiple myeloma (MM), medullary plasmacytoma (MP), and extramedullary plasmacytoma (EMP) [1, 2]. Extramedullary plasmacytoma is a rare entity and usually involves the nasopharynx or upper respiratory tract [3]. Involvement of the gastrointestinal tract (GIT) occurs in approximately $10 \%$ of EMP cases, with the stomach and small intestine being most commonly involved [1, 3-5]. Solitary EMP of the rectum is exceedingly rare [3].

We present a case of an 88-year-old male patient admitted to our clinic for signs and symptoms suggestive of ulcerative colitis relapse. He was diagnosed with ulcerative proctosigmoiditis 5 years earlier and treated with mesalazine. He reported bloody diarrhoea (approximately) 10 bowel movements/day, faecal incontinence, abdominal and back pain, weakness, and weight loss. Examination revealed cachexia, tachycardia, and abdominal tenderness. The patient's comorbidities included arterial hypertension, chronic obstructive pulmonary disease (COPD), type 2 diabetes, and gallstones. His family history was negative for haematological or gastrointestinal neoplasms. Previous colonoscopy was performed 3 years earlier, showing endoscopic and histological remission. On admission, laboratory analysis revealed microcytic anaemia, mild hypoproteinaemia and hypoalbuminaemia, elevated parameters of inflammation (C-reactive protein $83 \mathrm{mg} / \mathrm{l}$, erythrocyte sedimentation rate $38 \mathrm{~mm} / \mathrm{h}$ ), and elevated faecal calprotectin level $(1395 \mu \mathrm{g} / \mathrm{g})$. Plain chest radiography showed signs of COPD, and plain abdominal X-ray was normal. Stool was negative for Clostridium difficile toxin and cytomegalovirus. Upon admission the patient was started on topical and oral mesalazine therapy, antibiotics, and supportive care.
Colonoscopy was performed, revealing tumour-like, whitish, and hard (on biopsies) mass located in the proximal part of the anal canal, rectum, and rectosigmoid junction (Figure 1). Standard histopathology examination was suggestive of immunoproliferative disease, requiring further evaluation. Immunohistochemistry was performed and showed CD38+, $\kappa+, \lambda-$, MUM-1+, EBV-LMP-, CD20-, CD138-, and Ki 67+ in 60\% positive cells, indicating plasmablastic plasmacytoma (Figure 2). Further diagnostic algorithm excluded possible differential entities such as myeloma and lymphoma. Serum protein electrophoresis detected paraprotein, and immunofixation identified immunoglobulin $\mathrm{G}$ (IgG), $\lambda$ type. Urine protein electrophoresis did not indicate the presence of Bence-Jones protein. Alkaline phosphatase, calcium level, and lactate dehydrogenase were normal, and the concentration of $\beta 2$ microglobulin was elevated (13.2 mg/l, reference range: 0.9-3.0 mg/l). Sternal biopsy showed unspecific reactive bone marrow changes without elements of lymphoproliferative disorders. Multislice computed tomography (MSCT) showed a tumour mass $10 \times 11 \times 16 \mathrm{~cm}$ in size occupying the abdomen and pelvis, surrounding the rectum, iun the proximal part of anal canal, with parailiac adenopathy, ascites, and peritoneal involvement, and invading the urinary bladder and prostate (Figure 3 ). We additionally reviewed slides from previous biopsies, which surprisingly revealed monoclonality despite gross histopathology being normal. His performance status prevented him from receiving further oncological therapy. His condition progressively worsened, and he died 1 month after the diagnosis was established.

The cumulative incidence of solitary plasmacytoma was reported as $0.15 / 100,000$ [6]. Diagnosis of SP is made by histological and immunohistochemical analysis of tissue biopsy, and its main characteristic is the 

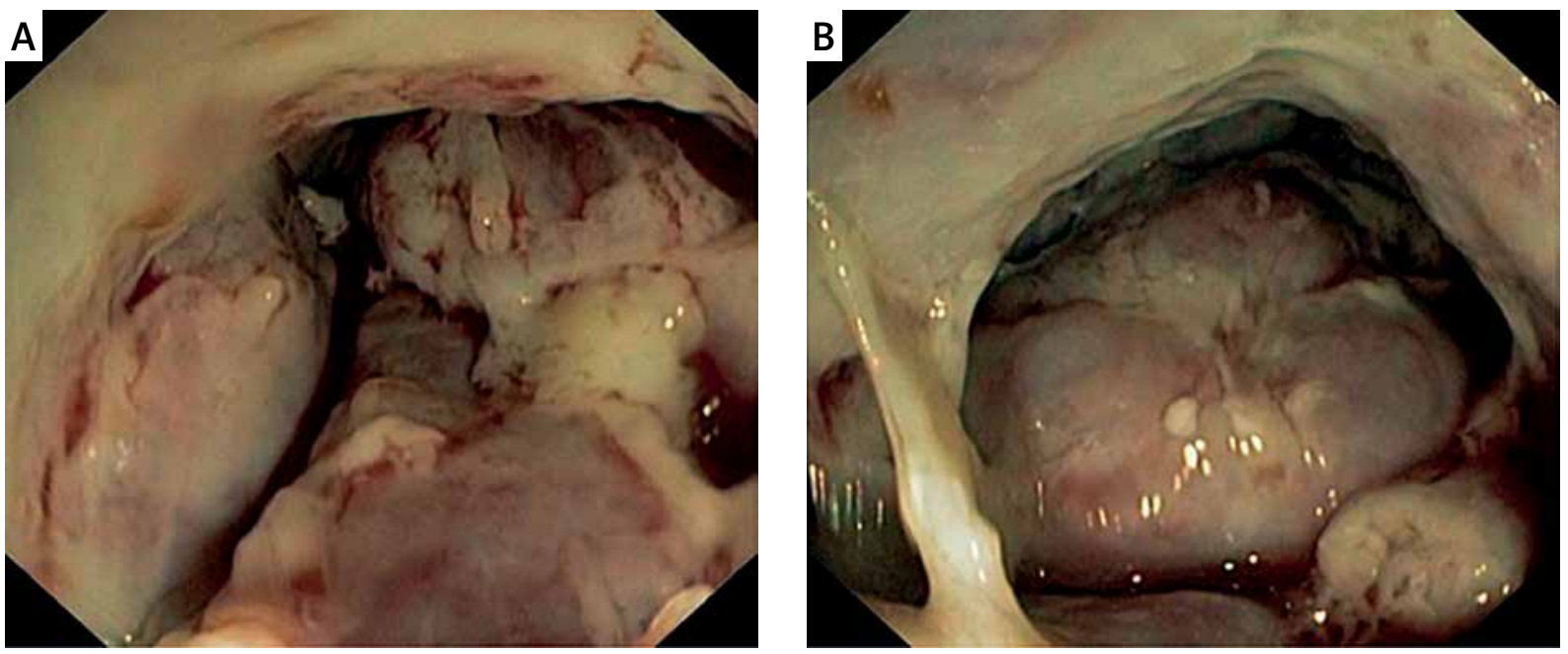

Figure 1. Gross appearance of a tumour mass located in the anal canal and rectum
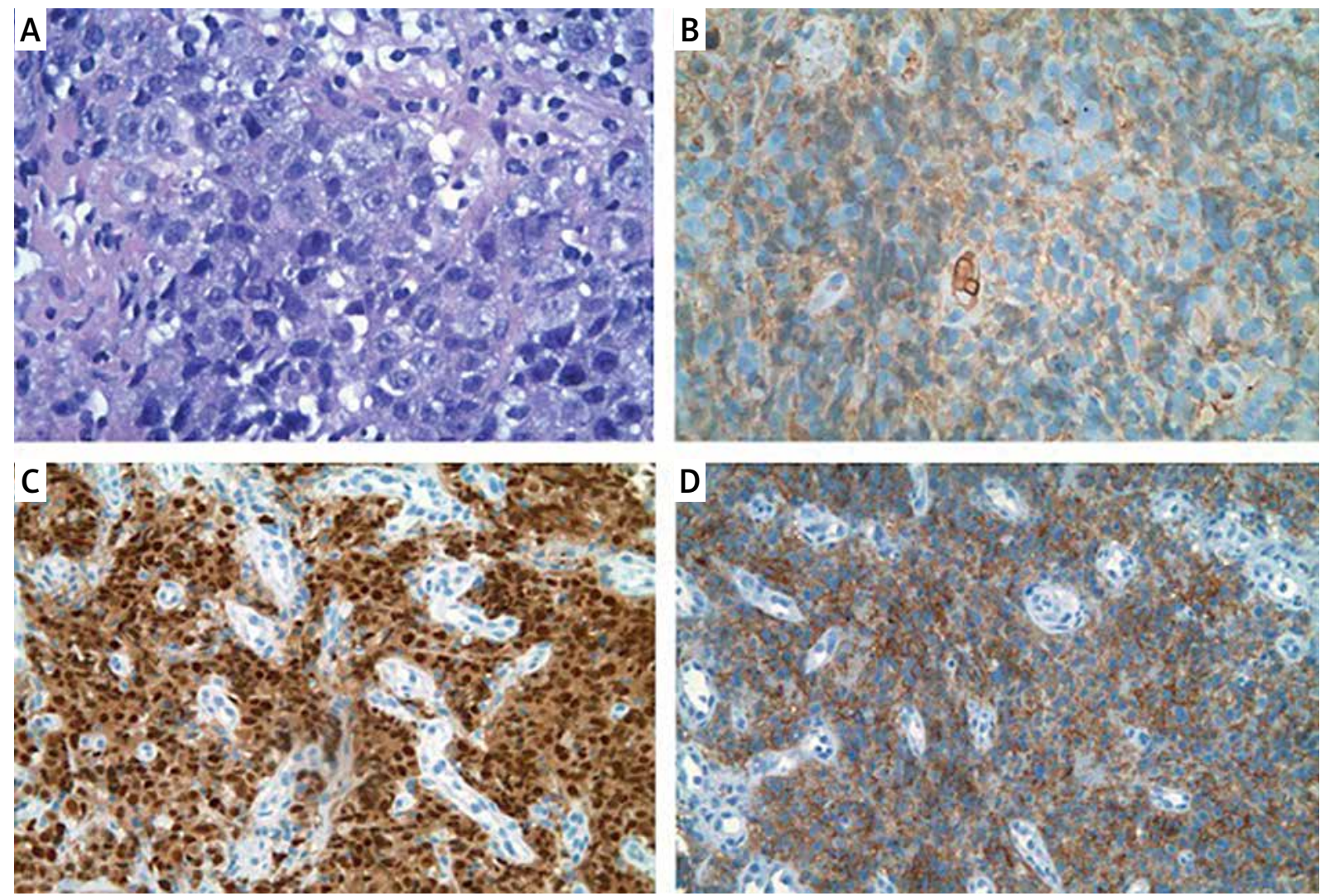

Figure 2. High magnification reveals that most of the neoplastic cells are plasmablasts with a prominent eosinophilic nucleolus (A). Immunohistochemistry for the specimens revealed Kappa+ (B), MUM1+ (C), and CD38+ (D). H\&E, 100x

presence of a homogenous infiltrate of monoclonal plasma cells positive for CD138 and/or CD38 [7]. Alexiou et al. reported that in $82.2 \%$ of cases EMP affected the nasopharynx and upper respiratory tract, which is in line with the result of $80 \%$ in the study by Galieni et al. [8]. The occurrence of extramedullary plasmacytoma in the rectum and anal canal is extremely rare. Literature regarding this rare condition is lacking and 


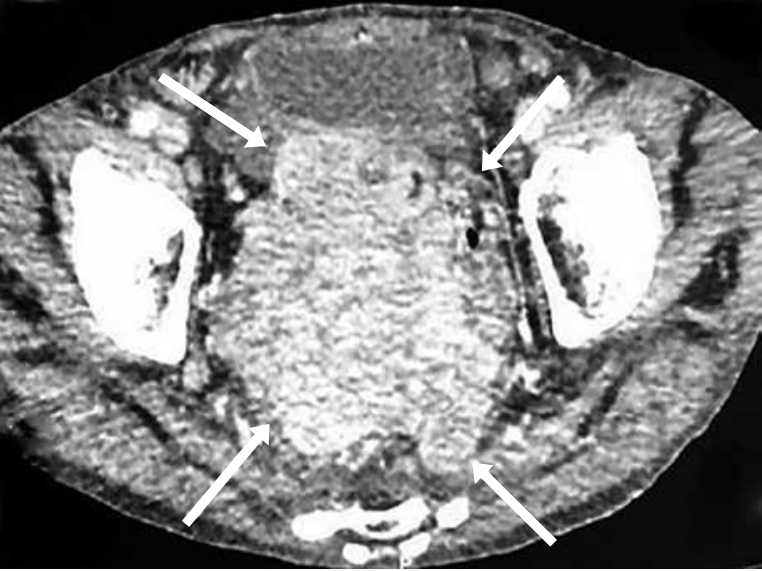

Figure 3. Multislice computed tomography axial scan of the abdomen revealed a tumour mass (arrows) $10 \times 11 \times 16 \mathrm{~cm}$ in size surrounding the rectum and the proximal part of anal canal with peritoneal involvement, invading the urinary bladder and prostate

mostly based on clinical reports. To our knowledge there are only 2 cases of EMP in the rectum and anal canal reported in patients with ulcerative colitis $[9,10]$.

Presenting symptoms are usually related to the site of plasma cell infiltration. In our case the patient claimed diarrhoea and blood in stool as well as faecal incontinence for the past 2 months. The endoscopic appearance of EMP arising from the gastrointestinal tract may vary, ranging from small sized polyp, as in the case reported previously by Miwa et al., to ulcerated masses or massive, whitish infiltration (as in the present case). Colonoscopy showed a massive tumour, which could be mistaken for adenocarcinoma or a neuroendocrine tumour (NET). Diagnosis of EMP must be supported by biopsy and immunohistochemistry following the exclusion of other possible diseases.

Our patient was treated with mesalazine only, and he had not taken any immunomodulatory therapy; thus, the possibility of these drugs playing a potential role in the development of EMP could not be considered in our case. Lee et al. reported a similar case of EMP presenting with abdominal pain and diarrhoea, but without previously diagnosed ulcerative colitis [1]. Given its clinical presentation, our patient could easily be mistaken for disease relapse. Additionally, it seems that EMP was developing in parallel to UC, but it is not clear whether it was initiated or worsened by chronic inflammation. Given the complexity, a multidisciplinary approach is needed in order to establish a correct diagnosis and therapeutic modality.
Treatment of EMPs varies - from surgery or chemotherapy to radiotherapy (RT) [11]. PCNs are highly sensitive to radiation, confirming that radiation therapy is the standard treatment for solitary bone plasmacytoma and EMP $[12,13]$. For patients with EMP, surgery might be able to resect large and well-defined masses but should be followed by radiotherapy [7]. On the other hand, it is not clear whether this would be the treatment of choice in the case of our patient due to an unfavourable event. His performance status prevented him from receiving further oncological therapy. His condition progressively worsened, and he died 1 month after the diagnosis was established.

EMP in patients with UC is extremely rare. Its existence could be sui generis or perhaps complicating a long-standing history of inflammatory bowel disease. Nevertheless, clinicians should be aware of this rare entity even in patients with confirmed previous diagnosis affecting the anorectum (e.g. ulcerative colitis).

\section{Conflict of interest}

The authors declare no conflict of interest.

\section{References}

1. Lee SH, Ahn BK, Baek SU, Chang HK. Primary isolated extramedullary plasmacytoma in the colon. Gastroenterology Res 2013; 6: 152-5.

2. Alexiou C, Kau RJ, Dietzfelbinger $\mathrm{H}$, et al. Extramedullary plasmacytoma: tumor occurrence and therapeutic concepts. Cancer 1999; 85: 2305-14

3. Gohil MH, Bhavsar DC, Suryanarayana U, Jetly DH. Plasmacytoma rectum extending to para-rectal region. J Can Res Ther 2015; 11: 662.

4. Hampton JM, Gandy JR. Plasmacytoma of the gastrointestinal tract. Ann Surg 1957; 145: 415-22.

5. Asselah F, Crow J, Slavin G, et al. Primary plasmacytoma of the small intestine. Histopathology 1982; 6: 631-45.

6. Dimopoulos MA, Moulopoulos LA, Maniatis A, Alexanian R. Solitary plasmacytoma of bone and asymptomatic multiple myeloma. Blood 2000; 96: 2037-44.

7. Caers J, Paiva B, Zamagni E, et al. Diagnosis, treatment, and response assessment in solitary plasmacytoma: updated recommendations from a European Expert Panel. J Hematol Oncol 2018; 11: 10.

8. Galieni P, Cavo M, Pulsoni A, et al. Clinical outcome of extramedullary plasmacytoma. Haematologica 2000; 85: 47-51.

9. Miwa W, Hiratsuka T, Tei S, et al. Solitary extramedullary plasmacytoma of the rectum complicating ulcerative colitis. Clin J Gastroenterol 2019; 12: 160-5.

10. Hashiguchi K, Iwai A, Inoue T, et al. Extramedullary plasmacytoma of the rectum arising in ulcerative colitis: case report and review. Gastrointest Endosc 2004; 59: 304-7.

11. Wen G, Wang W, Zhang Y, et al. Management of extramedullary plasmacytoma: role of radiotherapy and prognostic factor analysis in 55 patients. Chin J Cancer Res 2017; 29: 438-46. 
12. Kilciksiz S, Celik OK, Pak Y, et al. Clinical and prognostic features of plasmacytomas: a multicenter study of Turkish Oncol- ogy Group-Sarcoma Working Party. Am J Hematol 2008; 83: 702-7.

13. Soutar R, Lucraft $H$, Jackson $G$, et al. Guidelines on the diagnosis and management of solitary plasmacytoma of bone and solitary extramedullary plasmacytoma. Br J Haematol 2004; 124: 717-26.

Received: 23.03 .2020

Accepted: 8.09.2020 